Artículo científico

Volumen 32(3):719-732. Septiembre-diciembre, 2021 e-ISSN 2215-3608, doi:10.15517/am.v32i3.42303

https://revistas.ucr.ac.cr/index.php/agromeso/index

\title{
Evaluación agronómica y fisiológica en clones de camote (Ipomoea batatas) sometidos a condiciones de estrés hídrico ${ }^{1}$
}

\section{Agronomic and physiological evaluation in sweet potato (Ipomoea batatas) clones subjected to hydric stress conditions}

\author{
Rommel Igor León-Pacheco ${ }^{2}$, Mercedes Pérez-Macias ${ }^{3}$,Francia Concepción Fuenmayor-Campos ${ }^{3}$, Adrián José \\ Rodríguez-Izquierdo ${ }^{3}$, Gustavo Adolfo Rodríguez-Yzquierdo ${ }^{4}$, Edwin Andres Villagran-Munar ${ }^{4}$
}

1 Recepción: 18 de junio, 2020. Aceptación: 2 de marzo, 2021. Este trabajo formó parte de una investigación realizada en el Centro Nacional de Investigaciones Agropecuarias, Colombia.

2 Corporación Colombiana de Investigación Agropecuaria (AGROSAVIA). Zona bananera, Magdalena, Colombia. Apdo 478020. rleon@ agrosavia.co (autor para la correspondencia, https://orcid.org/0000-0002-9928-5282).

3 Instituto Nacional de Investigación Agrícola, Centro Nacional de Investigación Agropecuaria. Maracay, Venezuela. Apdo 2101. maciasmercedes@yahoo.com (https://orcid.org/0000-0002-2497-0031); ffuenmayorc@gmail.com (https://orcid.org/0000-0003-4732-3096); adrianjri65@hotmail.com (https://orcid.org/0000-0002-2270-7475).

4 Corporación Colombiana de Investigación Agropecuaria (AGROSAVIA). Mosquera, Cundinamarca, Colombia. Apdo 344300. grodriguezy@ agrosavia.co (https://orcid.org/0000-0003-3709-8534). evillagram@agrosavia.co (https://orcid.org/0000-0003-1860-5932).

\section{Resumen}

Introducción. Las raíces y tubérculos como el camote (Ipomoea batatas L.) se encuentran entre los seis primeros cultivos más importantes del mundo. Sin embargo, esta especie es sensible al estrés por déficit hídrico. El uso de genotipos resistentes a la sequía y mejores prácticas de gestión del agua pueden mejorar la calidad y el rendimiento de la raíz. Para ampliar la productividad en tales ambientes se pueden utilizar prácticas de riego complementario o identificar genotipos con potencial de rendimiento promisorios frente a estas condiciones. Objetivo. Evaluar el efecto del estrés hídrico en variables fisiológicas y agronómicas en clones de camote. Materiales y métodos. Se realizó un ensayo en el período de abril-julio 2015 en el Centro Nacional de Investigación Agropecuaria, Maracay, Venezuela, con tres repeticiones bajo un diseño en parcelas divididas, donde la parcela principal estuvo conformada por tres niveles de riego (todo el ciclo, dos y tres primeros meses con riego) y la parcela secundaria por tres clones de camote. Se midieron variables asociadas al intercambio gaseoso, crecimiento vegetativo, biomasa y rendimiento. Resultados. Las plantas de camote frente a condiciones de sequía disminuyeron su crecimiento, expansión foliar, cerraron en forma parcial los estomas para evitar pérdidas de agua por transpiración sin afectar las variables de tasa neta de asimilación de $\mathrm{CO}_{2}$, rendimiento y acumulación de biomasa. El mejor clon fue el 64, con mayor transpiración, acumulación de biomasa y rendimiento de 18,3 $\mathrm{t} \mathrm{ha}^{-1}$. El riego en todo el cultivo generó el mayor crecimiento vegetativo con 125 hojas planta $^{-1}$. Conclusiones. Bajo condiciones de estrés hídrico las plantas de camote se adaptaron fisiológicamente para no perder agua por la transpiración sin sacrificar las tasas de fotosíntesis, con las cuales los clones lograron alcanzar una acumulación de biomasa aérea, raíces y rendimiento del tubérculo similar al cultivo con riego.

Palabras clave: crecimiento, intercambio gaseoso, productividad, sequía. 


\begin{abstract}
Introduction. Roots and tubers, such as sweet potatoes (Ipomoea batatas L.), are among the top six most important crops worldwide. However, this species is sensitive to stress due to water deficit. The use of drought resistant genotypes and better water management practices can enhance its' root quality and yield. In order to enhance productivity in those environments, complementary irrigation practices can be used or genotypes with promising yield potential against these conditions can be identified. Objective. To evaluate the effect of water stress on physiological and agronomic variables in sweet potato clones. Materials and methods. A trial was conducted from April to July 2015 at the National Center for Agricultural Research, Maracay, Venezuela, with three replications under a split plot design, where the principal plot was integrated by three irrigation treatments (all cycles, two, and three first months with irrigation) and the secondary plot by three sweet potatoes clones. Variables associated with gas exchange, vegetative growth, biomass, and yield were measured. Results. The results show that sweet potato plants against drought conditions decreased their growth, foliar expansion, partially closed the stomata avoiding water losses through transpiration without affecting the variables of net assimilation rate of $\mathrm{CO}_{2}$, yield, and biomass accumulation. The best clone with increased transpiration, biomass accumulation, and yield of $18.3 \mathrm{t} \mathrm{ha}^{-1}$ was the 64. Irrigation throughout the crop cycle generated the highest vegetative growth with 125 leaves plant ${ }^{-1}$. Conclusions. Under water stress conditions, sweet potato plants adapted physiologically to avoid water loss through transpiration without sacrificing photosynthetic rates, with which the clones were able to accumulate aerial biomass, roots, and tuber yield similar to the irrigated crop.
\end{abstract}

Keywords: growth, gas exchange, productivity, drought.

\title{
Introducción
}

La batata o camote (Ipomoea batatas L.) es muy empleado en la alimentación humana, tanto en fresco o mediante la transformación industrial en diversos productos como alimentos concentrados para animales, pastelería y repostería, incluso para la obtención de bebidas alcohólicas, dada su riqueza en sustancias amiláceas y azucaradas. Además, es una fuente de carbohidratos, vitamina A, vitamina C, proteínas y tiene una importancia sociocultural y en términos de seguridad agroalimentaria (Omotobora et al., 2014; Yooyongwech et al., 2013). Es un cultivo que presenta escasas exigencias edafoclimáticas y es tolerante a factores bióticos (Muhammad et al., 2011).

La batata es una de las raíces y tubérculos más importante del mundo con una superficie cosechada de 9202 777 ha y un rendimiento promedio de 12,26 t ha ${ }^{-1}$ (Food and Agriculture Organization, 2019); en Venezuela es la quinta raíz más importante del país con 1947 ha y rendimiento promedio de 9,413 t ha-1 (Confederación de Asociaciones de Productores Agropecuarios de Venezuela, 2019).

El camote es un cultivo de bajo costo de producción, que se produce casi todo el año en países en vías de desarrollo. En regiones áridas y semiáridas, la presencia de estrés hídrico y salino puede generar disminución de los rendimientos y pérdidas en la calidad de las raíces tuberosas (Omotobora et al., 2014; Yooyongwech et al., 2013; Zhang et al., 2015).

A pesar que el cultivo es tolerante a la sequía, las plantas de camote no toleran períodos de déficit hídrico durante la siembra inicial y durante las etapas iniciales de crecimiento, incluido el desarrollo y la iniciación del tubérculo (Ciryl et al., 2015), por lo que es necesario identificar genotipos apropiados adaptados a las condiciones de sequía. Una gran alternativa que tiene el cultivo para producir frente a estas condiciones es la alta variabilidad genética, con la cual se pueden identificar cultivares adaptados a las condiciones con estos tipos de estres (Laurie et al., 2015). 
El riego suplementario para el camote es recomendable cuando la humedad disponible del suelo es inferior al $20 \%$ (Ravi \& Indira, 1999) y láminas de agua del $60 \%$ del nivel de agotamiento de humedad, lo que podría aumentar el rendimiento de la raíz en plantas de camote en un $24 \%$ (Mukhopadhyay et al., 2011). La compensación entre rendimiento y supervivencia al estrés hídrico también está relacionada con características fisiológicas y cambios bioquímicos en las hojas. Bajo déficit hídrico, la resistencia estomática tiende a aumentar para preservar el contenido de agua de la hoja y prevenir la senescencia de la misma, sin embargo, incrementos de la resistencia estomática, también disminuyen el intercambio de $\mathrm{CO}_{2}$, la tasa fotosintética neta y el rendimiento. Por lo tanto, si se producen sequías durante el inicio de la tuberización procesos fisiológicos de este tipo podrían reducir el rendimiento (Mukhopadhyay et al., 2011). Este mismo comportamiento lo observaron Yooyongwech et al. (2014) quienes reportaron las interacciones entre clones y tratamientos de déficit hídrico con menores tasas de fotosíntesis, transpiración y conductancia estomáticas, valores que oscilaron entre $0,87-5,35 \mu \mathrm{mol} \mathrm{CO} \mathrm{m}^{-2} \mathrm{~s}^{-1}$; $3,03-20,28 \mathrm{mmol} \mathrm{CO}_{2} \mathrm{~m}^{-2} \mathrm{~s}^{-1}$ y $0,16-0,38 \mathrm{~mol} \mathrm{H}_{2} \mathrm{O} \mathrm{m}^{-2} \mathrm{~s}^{-1}$, respectivamente, para los tratamientos con estrés severo ( $8 \%$ de la humedad del suelo) en comparación con los tratamientos irrigados con valores diez veces mayores que los tratamientos estresados.

En Sudáfrica, cincuenta accesiones de camote se plantaron para su evaluación bajo condiciones de sequía (retención de la aplicación de las láminas de agua) en invernadero, registró los valores más altos de número de hojas en el clon de camote zapallo (40-60 hojas), con los valores más bajos (22 y 14 hojas) para dos ciclos de evaluación del 2004, seguido por el clon Ejumula con 37 hojas y el comportamiento menos promisorio con el clon Phala (Omotobora et al., 2014). El estrés por sequía disminuye significativamente el contenido relativo de agua (RWC), lo que tiene un fuerte efecto en la tasa fotosintética y esto también conduce a un aumento en la temperatura de la hoja y el dosel (Siddique et al., 2000).

El suministro de agua a través del riego complementario es muy importante en las dos primeras semanas después del trasplante para favorecer el enraizamiento de las plántulas recién sembradas. Trabajos realizados por el Instituto Nacional de Tecnología Agropecuaria (INTA) en Argentina han observado reducciones de 80 a $90 \%$ en el rendimiento por estrés de humedad al inicio del proceso de formación de las raíces reservantes, es decir, durante el primer y segundo mes después de la plantación. Esta fase coincide con la ocurrencia de precipitaciones en casi todas las zonas productoras. En ausencia de las mismas, se debe aplicar una lámina de riego de 30-40 mm hasta que la situación de estrés hídrico desaparezca (Cusumano \& Zamudio, 2013).

Diferentes investigaciones permitieron explicar el efecto del estrés hídrico sobre el control estomático del cultivo de camote sobre variables asociadas al rendimiento, entre ellos, el trabajo de Zhang et al. (2015), en el que observaron que la distribución de materia seca y el intercambio de gases en el cultivo aumentó con el incremento del contenido de agua en el suelo, con valores de conductancia estomática y tasa de fotosíntesis con rangos de 56$206 \mathrm{mmol} \mathrm{CO} \mathrm{m}^{-2} \mathrm{~s}^{-1}$ y 3-11 $\mu \mathrm{mol} \mathrm{CO} \mathrm{m}^{-2} \mathrm{~s}^{-1}$, mientras que Guerrero (2020) mostró una reducción promedio de $79,56 \pm 23,12$ por ciento en la variable de conductancia estomática comparado con las plantas no estresadas. Este comportamiento fue explicado en una investigación realizada en Perú, con genotipos de parientes silvestres de camotes, en el que se demostró una clara diversidad en su respuesta fisiológica ante la inducción de memoria para desarrollar tolerancia al estrés hídrico (Guerrero, 2020). Asimismo, La Rosa (2008), observó las altas resistencias estomáticas por algunos cultivares de camote.

Diferencias para el rendimiento en clones de camote fueron observadas en el trabajo de Omotobora et al. (2014), sobresaliendo el clon Tacna $\left(9,24 \mathrm{t} \mathrm{ha}^{-1}\right)$ y Zapallo $\left(6,16 \mathrm{t} \mathrm{ha}^{-1}\right)$. Estos valores de rendimiento son comparables a los registrados en el cultivo de camote sembrado en condiciones de subsistencia de secano (5-10

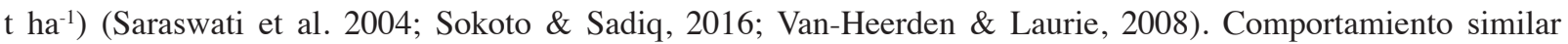
reportaron Mantovani et al. (2013), quienes encontraron que la diferencia de productividad entre los cultivares fue del $25,8 \%$, con un aumento en el consumo de agua (21,5 $\mathrm{mm}$ con 6,2\% más que el resto) para el clon que tuvo los mayores rendimientos. Los rendimientos en el cultivo de camote los afectó la restricción de agua y la salinidad. 
El cultivar comercial de Perú Untacip superior, tuvo un porcentaje de pérdida de rendimiento en condiciones de salinidad mayor que en el cultivar Huambachero. En el mismo estudio se encontró que el estrés hídrico y salino causó una menor extracción de nutrientes (Rodríguez, 2016). La disminución del estrés hídrico aplicado, resultó en un aumento en el rendimiento de las raíces tuberosas de ambos cultivares de camote; sin embargo, este aumento no fue lineal, alcanzando una productividad de 49,8 $\mathrm{t} \mathrm{ha}^{-1}$ con la aplicación de 325,5 mm y de 67,1 t ha ${ }^{-1}$ con 347 $\mathrm{mm}$ (Mantovani et al., 2013). Las diferencias genotípicas y las interacciones de los genotipos por el estrés fueron significativas para el rendimiento de raíz. El genotipo menos estresado por agua con el menor potencial hídrico tuvo el mayor rendimiento de raíz, entre tanto, los genotipos de camote más resistentes a la sequía fueron los que tuvieron los mayores rendimientos de raíz con valores de 0,92 a 10,4 t ha ${ }^{-1}$ (Ekanayake \& Collins, 2004).

El estrés hídrico y salino provocan una respuesta fisiológica que afecta el rendimiento del camote, en ese sentido, trabajos realizados en Cuba y Perú permitieron observar dos mecanismos de tolerancia, escape de la sequía (senescencia tardía y aumento del área foliar) en accesiones de floración temprana y evitación de la sequía (optimización del intercambio gaseoso y transpiración de las hojas) en accesiones con floración tardía (Guerrero, 2020; Rodríguez, 2016). A pesar de ello, en Latinoamérica poco ha sido el esfuerzo por profundizar más en la respuesta al estrés hídrico en el cultivo de camote.

El objetivo de este trabajo fue evaluar en clones de camote el efecto del estrés hídrico en variables fisiológicas y agronómicas.

\section{Materiales y métodos}

\section{Características generales del área de estudio}

El estudio se realizó en el Centro Nacional de Investigaciones Agropecuarias, Campo Experimental del Instituto Nacional de Investigaciones Agrícolas (INIA-CENIAP), Maracay, estado Aragua, Venezuela; ubicado en zona de Bosque Seco Tropical Premontano, 10¹7’14” N y 67³6’02” O, altura 480 msnm.

El clima de la zona está clasificado como Bosque Seco Tropical, con un promedio anual de radiación global de 18,5 $\mathrm{Mjm}^{2} \mathrm{~d}^{-1}$ con valores máximos de 20,7 $\mathrm{Mjm}^{2} \mathrm{~d}^{-1}$ en marzo y mínimos de $17,2 \mathrm{Mjm}^{2} \mathrm{~d}^{-1}$ en noviembre.

La fecha de siembra fue abril del 2015 con precipitación durante todo el ciclo del cultivo de 195,6 mm (abriljulio 2015), valores muy por debajo de la evapotranspiración del cultivo $(724,00 \mathrm{~mm})$, motivado a que la siembra estuvo dispuesta en época seca durante su fase de crecimiento vegetativo. La temperatura varió entre $20-30{ }^{\circ} \mathrm{C}$, con humedad relativa entre $60-90 \%$ (Figura 1).

El cultivo se sembró en un lote con suelo de textura franco-limosa, cuyo análisis químico mostró valores de 57 $\mathrm{mg} \mathrm{kg}{ }^{-1}$ de fósforo; $111 \mathrm{mg} \mathrm{kg}^{-1}$ de potasio; $600 \mathrm{mg} \mathrm{kg}^{-1}$ de calcio; $21 \mathrm{mg} \mathrm{kg}^{-1}$ de magnesio; 2,76 \% de materia orgánica; 6,1 de $\mathrm{pH}$ y conductividad hidráulica de $0,13 \mathrm{~d} \mathrm{~m}^{-1}$ (unidad de servicio de análisis de suelo-agua-planta del CENIAP).

El suelo se preparó con tres pases de rastrillas, con el fin de dejar el suelo con una profundidad aproximada de $30 \mathrm{~cm}$, y un pase de surcado (surcos de $40 \mathrm{~cm}$ de alto). La siembra fue directa en campo con esquejes de la parte apical con 7-8 yemas.

\section{Material vegetal}

Se estudiaron tres clones promisorios de camote (Ipomoea batatas L.) del banco de germoplasma del Instituto Nacional de Investigaciones Agrícolas, Centro Nacional de Investigaciones Agropecuarias (INIA-CENIAP), Maracay, estado Aragua, Venezuela. El clon '2894' con un tipo de planta semierecta, entrenudo de tallo intermedio, color de follaje verde, raíz larga-curvada, color de raíz crema; el clon '2878' con un tipo de planta dispersa, raíz 


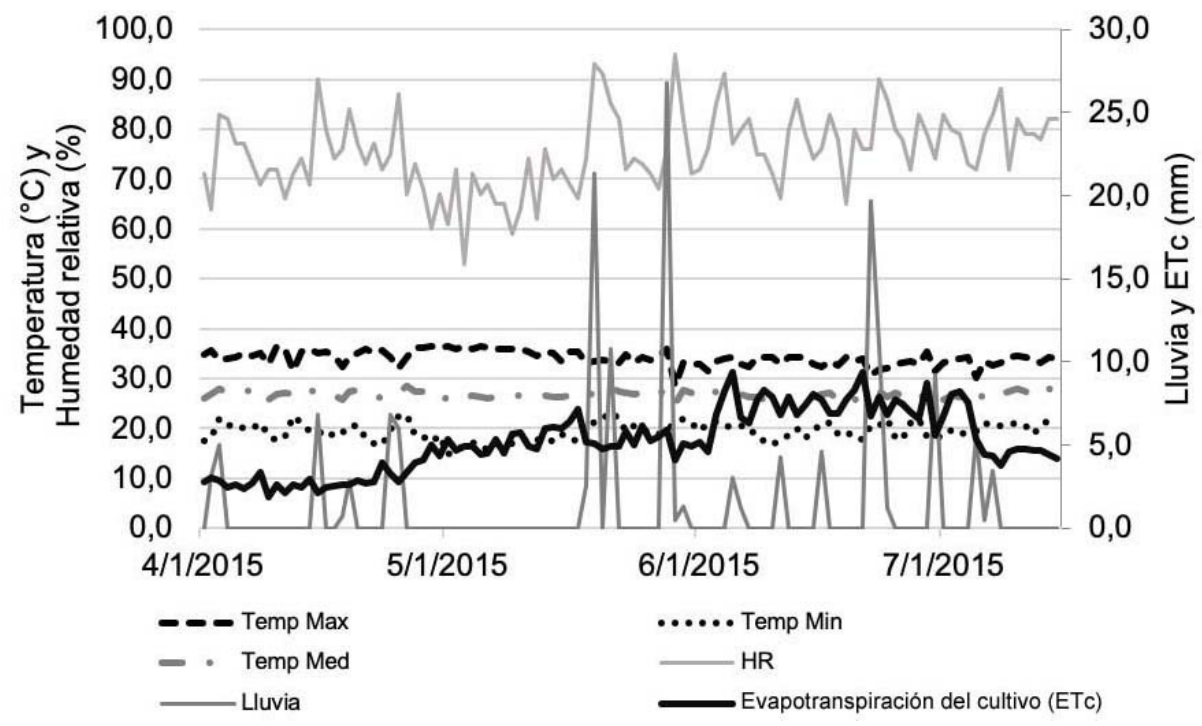

Figura 1. Climograma de abril-julio 2015 en el Instituto Nacional de Investigaciones Agrícolas-Centro Nacional de Investigaciones Agropecuarias (INIA-CENIAP), Maracay, Venezuela.

ETc: evapotranspiración del cultivo.

Figure 1. Climograph of April-July 2015 at the Instituto Nacional de Investigaciones Agrícolas-Centro Nacional de Investigaciones Agropecuarias (INIA-CENIAP), Maracay, Venezuela.

ETc: crop evapotranspiration.

redonda, color de raíz blanca y el clon '64' con un tipo de planta erecta, color de follaje verde con nervaduras moradas, raíz redonda-eliptica, color de raíz amarillo oscuro. Los clones se seleccionaron en función de su comportamiento potencial favorable frente a la sequía.

\section{Descripción del experimento}

Se sembraron tres surcos de $5 \mathrm{~m}$ de largo con una densidad de siembra de 0,25 metros entre plantas y $1 \mathrm{~m}$ entre surco (32000 plantas ha-1), para un área total por unidad experimental de $25 \mathrm{~m}^{2}$, correspondiente a cinco surcos separados a $1 \mathrm{~m}$ cada uno y $5 \mathrm{~m}$ de largo, respectivamente. El riego utilizado fue por goteo, con un tanque de 2000 $\mathrm{L}$ de capacidad y bomba de $1 \mathrm{hp}$, con cintas de goteo con descarga de $0,71 \mathrm{~h}^{-1}$ y frecuencias de riego de dos veces por semana hasta mantener el suelo a capacidad de campo (10-20 centibares). Para estimar el potencial de agua del suelo se utilizó tensiómetro de suelo marca Irrometer. El manejo agronómico fue homogéneo para todos los tratamientos durante todo el ciclo del cultivo, se realizó control de malezas manual, no hubo incidencia de plagas y enfermedades durante el ciclo de evaluación y la fertilización fue con $120 \mathrm{~kg} \mathrm{ha}^{-1}$ de nitrógeno (se usó como fuente urea) y $90 \mathrm{~kg} \mathrm{ha}^{-1}$ de fósforo [como fuente se utilizó fosfato diamónico (18-46-0)] (Cusumano \& Zamudio, 2013).

\section{Diseño del experimento}

Se aplicó un diseño en parcelas divididas, con tres repeticiones, la parcela principal estuvo conformada por manejo del riego y la franja secundaria por clones. Los factores clones ('2894', '2878' y '64”) y tratamientos de riego [control, riego durante todo el ciclo del cultivo (SE), con riego durante los primeros dos meses después 
de la siembra (CR2) y con riego los primeros tres meses después de la siembra (CR3)], se aleatorizaron en tres repeticiones. Cada unidad experimental tuvo una área total de $25 \mathrm{~m}^{2}$ y 80 plantas. El riego en los tratamientos con irrigación se hizo con espacios de tiempo semanal.

\section{Variables fisiológicas}

Grado de verdor (unidad Spad): se evaluó con el medidor de clorofila Spad 502 Konica Minolta. Se tomaron dos plantas y dos hojas planta ${ }^{-1}$ de la quinta hoja expandida en sentido ápice base, por cada unidad experimental al cuarto mes de medición.

Fotosíntesis $\left(\mu \mathrm{mol} \mathrm{CO}_{2} \mathrm{~m}^{-2} \mathrm{~s}^{-1}\right)$, conductancia estomática $\left(\mathrm{mmol} \mathrm{CO}_{2} \mathrm{~m}^{-2} \mathrm{~s}^{-1}\right)$ y transpiración $\left(\mathrm{mmol} \mathrm{H}_{2} \mathrm{O} \mathrm{m}^{-2} \mathrm{~s}^{-1}\right)$ : estas variables se midieron en las horas comprendidas desde las 10:00 hasta las 13:00 (De-Tafur, 2002), con un analizador infrarrojo de gases CI-340 Photosynthesis Systems, en dos hojas planta ${ }^{-1}$, de la quinta hoja totalmente expandida en sentido ápice base para cada unidad experimental al cuarto mes de medición.

\section{Variables de crecimiento}

Número de hojas: se contó el número de hojas de cuatro plantas del surco central por unidad experimental, desde la parte basal de la planta hasta la apical del tallo al cuarto mes de edad del cultivo.

Longitud del tallo $(\mathrm{cm})$ : se midió la altura de cuatro plantas del surco central por unidad experimental desde la parte basal de la planta hasta la parte apical del tallo central al cuarto mes de edad del cultivo

Peso fresco y seco de follaje y raíz (g): en cada unidad experimental se cosecharon diez plantas del surco central al quinto mes y se determinó el peso de raíces totales y el del follaje por planta.

\section{Análisis de datos}

Previa comprobación por el análisis de la varianza, se empleó el modelo lineal aditivo de un diseño en parcelas divididas y cuando se encontró diferencias entre promedios, se aplicó la prueba de comparación de medias de Tukey, con un nivel de significancia del $5 \%$. Se realizó un análisis multivariado por componentes principales con el programa Infostat, versión estudiantil.

\section{Resultados}

\section{Interacción entre los factores estrés hídrico por clon}

La longitud de la guía o bejuco fue la única variable con diferencias significativas $(\mathrm{p}<0,05)$ para la interacción estrés por clon (Cuadro 1). Las interacciones con mayor crecimiento para esta variable fueron los tratamientos de riego durante todo el ciclo del cultivo y riego durante los primeros tres meses con el clon 2894 con valores de 193,89 y 167,44 cm, respectivamente, lo que implica una gran importancia para la alimentación animal. Este clon se caracteriza por tener esquejes largos y fue el cultivar con la mayor longitud de la guía, independiente del estrés que se generó, lo que da indicios que la genética de los clones es lo que más afecta la interacción. Mientras que, los otros grupos de estres con clones alcanzaron valores de longitud de semilla de 98,33 a 50,78 cm, muy por debajo de los resultados obtenidos con el clon 2894 con los tres tratamientos de estrés.

Las variables fisiológicas de fotosíntesis, conductancia estomática y rendimiento no variaron significativamente $(p>0,05)$, pero el clon 64 alcanzó las mayores tasas de fotosíntesis en todos los tratamientos de estrés hídricos 
Cuadro 1. Longitud promedio del tallo (bejuco o guía) en tres clores de camote (Ipomoea batatas L.), cultivados con en tres niveles de estrés hídrico. Instituto Nacional de Investigaciones Agrícolas-Centro Nacional de Investigaciones Agropecuarias (INIA-CENIAP). Maracay, Venezuela, 2015.

Table 1. Stem length (vine or guide) average in three sweet potato (Ipomoea batatas L.) clones, cultivated with three water stress levels. Instituto Nacional de Investigaciones Agrícolas-Centro Nacional de Investigaciones Agropecuarias (INIA-CENIAP). Maracay, Venezuela, 2015.

\begin{tabular}{cccc}
\hline Estrés & Clon & DMS $=\mathbf{6 8 , 5 8 3 1 9}$ & \\
\hline SE & 2894 & $193,89 \pm 52,55$ & $\mathrm{~A}$ \\
CR3 & 2894 & $167,44 \pm 51,37$ & $\mathrm{~A}$ \\
CR2 & 2894 & $98,33 \pm 15,89$ & $\mathrm{~B}$ \\
$\mathrm{SE}$ & 2878 & $86,44 \pm 35,94$ & $\mathrm{~B}$ \\
$\mathrm{CR} 3$ & 2878 & $77,33 \pm 19,00$ & $\mathrm{~B}$ \\
$\mathrm{CR} 2$ & 2878 & $50,78 \pm 0,83$ & $\mathrm{~B}$ \\
$\mathrm{CR} 3$ & 64 & $59,67 \pm 2,18$ & $\mathrm{~B}$ \\
$\mathrm{SE}$ & 64 & $57,89 \pm 22,11$ & $\mathrm{~B}$ \\
$\mathrm{CR} 2$ & 64 & $57,44 \pm 10,19$ & $\mathrm{~B}$ \\
\hline
\end{tabular}

Medias con una letra común no son significativamente diferentes $(\mathrm{p}>0,05)$. / Means with a common letter are not significantly different (Tukey, $\mathrm{p}>0.05$ ).

Promedio \pm desviación estándar / Average \pm standard deviation.

con valores entre 15,70 y $17,22 \mu \mathrm{mol} \mathrm{CO} \mathrm{m}^{-2} \mathrm{~s}^{-1}$, lo que provocó los valores más altos para el peso aéreo $(520,83$ $\left.633,33 \mathrm{~g} \mathrm{planta}^{-1}\right)$ y para raíces totales $\left(479,16-654,16 \mathrm{~g} \mathrm{planta}^{-1}\right)$; y este clon fue el que tuvo comportamiento superior cuando se suspendió el riego después de los dos primeros meses después de la siembra (CR2) (Figura 1). Mientras que, el clon 2894 cuando fue regado durante todo el ciclo (SE) las plantas alcanzaron las mayores tasas de fotosíntesis y, por ende, el mejor comportamiento para las variables peso aéreo y raíz (Figura 2).

El clon 2894 sin estrés tuvo las plantas con los tallos más largos y mayor número de hojas, lo que provocó una mayor producción de follaje, comportamiento contrario al resto de los tratamientos (Figura 3). El clon 64 en todos los niveles de estrés mostró el mejor comportamiento agronómico durante todo el ciclo de evaluación, es así como se pudo encontrar relaciones importantes para las variables transpiración, conductancia estomática y peso seco y fresco de raíz (Figura 3). Todos los tratamientos de estrés con los clones 2894 y 2878, estuvieron relacionados en un grupo (Figura 3), con comportamiento opuesto a las combinaciones de los tratamientos de estrés con el clon 64 y estas con el grado de verdor, con la tendencia que todos los tratamientos de estrés durante todos los ciclos de evaluación alcanzaron el comportamiento agronómico menos promisorio (Figura 3).

\section{Efecto de los clones}

En el factor de los clones hubo diferencias significativas $(\mathrm{p} \leq 0,05)$ en la mayoría de las variables, destacándose el peso seco de raíz, transpiración, grado de verdor y peso de raíces totales. El clon 64 fue el que acumuló la mayor biomasa seca y fresca de raíz (PSR y PRT) con 261,11 g planta ${ }^{-1}$ y 572,22 $\mathrm{g} \mathrm{planta}^{-1}$, respectivamente, además tuvo las mayores tasas transpiratorias $\left(0,43 \mathrm{mmol} \mathrm{H}_{2} \mathrm{O} \mathrm{m}^{-2} \mathrm{~s}^{-1}\right)$, lo que provocó las mayores conductancias estomáticas y, por ende, mayores tasas de fotosíntesis. El mismo comportamiento se alcanzó en la parte vegetativa con área foliar de hasta el doble en comparación con el resto de los clones (Cuadro 2). Sin embargo, el grado de verdor, a pesar de estar asociado al contenido de clorofila, mostró los menores valores para el clon 64 (Cuadro 2). 

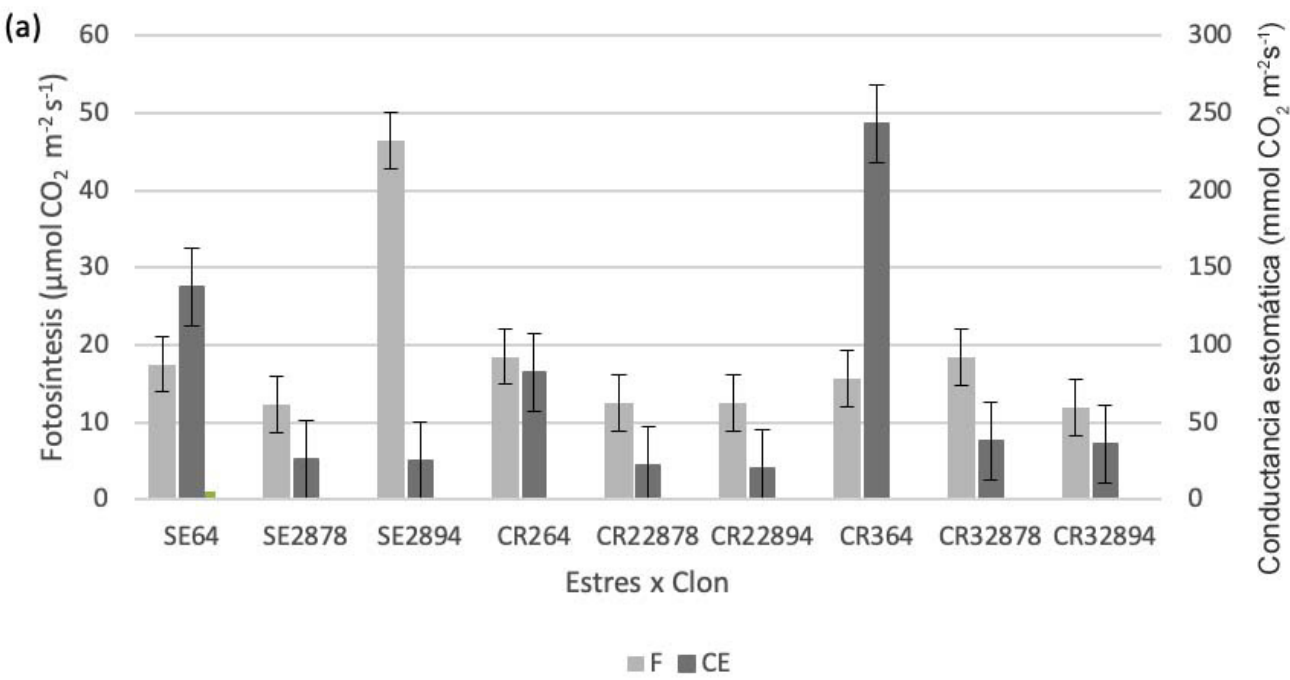

(b)

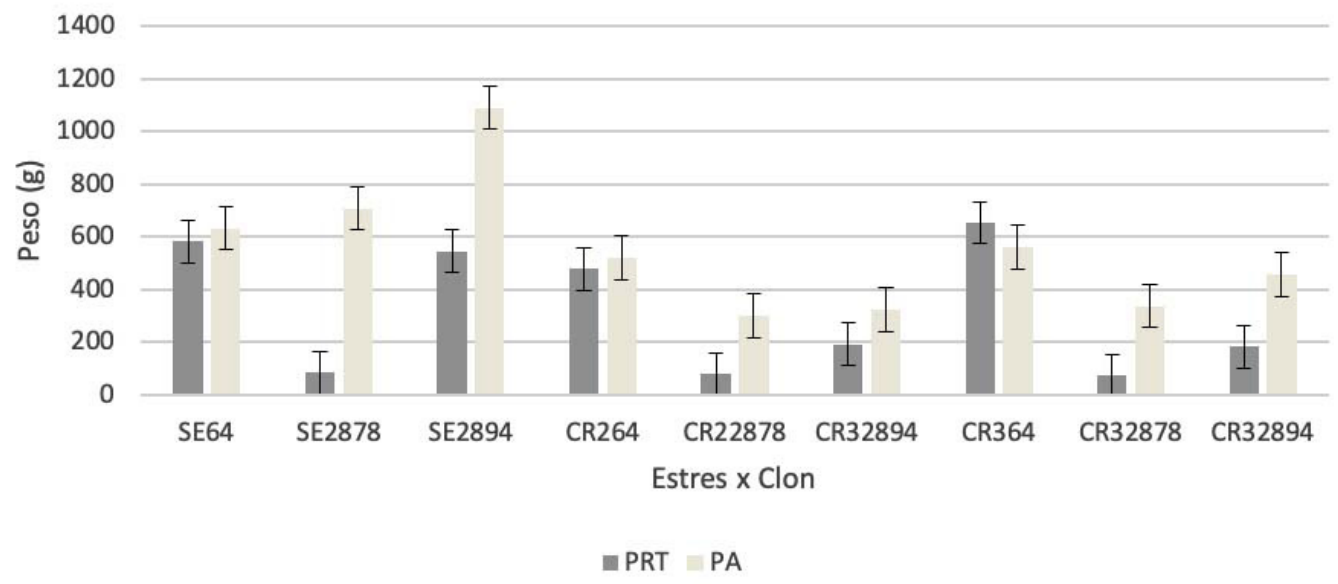

Figura 2. Valores promedio de fotosíntesis $(\mathrm{F})$, conductancia estomática (CE), peso aéreo (PA) y peso de raíces totales (PRT) de tres clones de camote (Ipomoea batatas L.) en tres tratamientos de estrés hídrico. Instituto Nacional de Investigaciones Agrícolas-Centro Nacional de Investigaciones Agropecuarias (INIA-CENIAP). Maracay, Venezuela, 2015.

SE: Riego durante todo el ciclo del cultivo, CR2: riego durante los primeros dos meses después de la siembra, CR3: riego durante los primeros tres meses después de la siembra. La barra corresponde a la desviación estándar.

Figure 2. Average values of photosynthesis (F), stomatal conductance (CE), above-ground weight (PA), and total root weight (PRT) of three clones of sweet potato (Ipomoea batatas L.) in three treatments of hydric stress. Instituto Nacional de Investigaciones AgrícolasCentro Nacional de Investigaciones Agropecuarias (INIA-CENIAP). Maracay, Venezuela, 2015.

SE: Irrigation throughout the whole crop cycle, CR2: irrigation during the first two months after sowing, CR3: irrigation during the first three months after sowing. The bar is equivalent to the standard deviation.

\section{Efecto de los tratamientos de estrés hídricos}

La única variable que cambió significativamente $(\mathrm{p} \leq 0,05)$ fue el número de hojas, destacándose el riego durante todo el ciclo del cultivo con 125 hojas, seguido por los tratamientos con riego por tres meses y con riego por dos meses, con valores de 57 y 47 hojas, respectivamente. Este comportamiento da evidencia que, para dedicarse a 


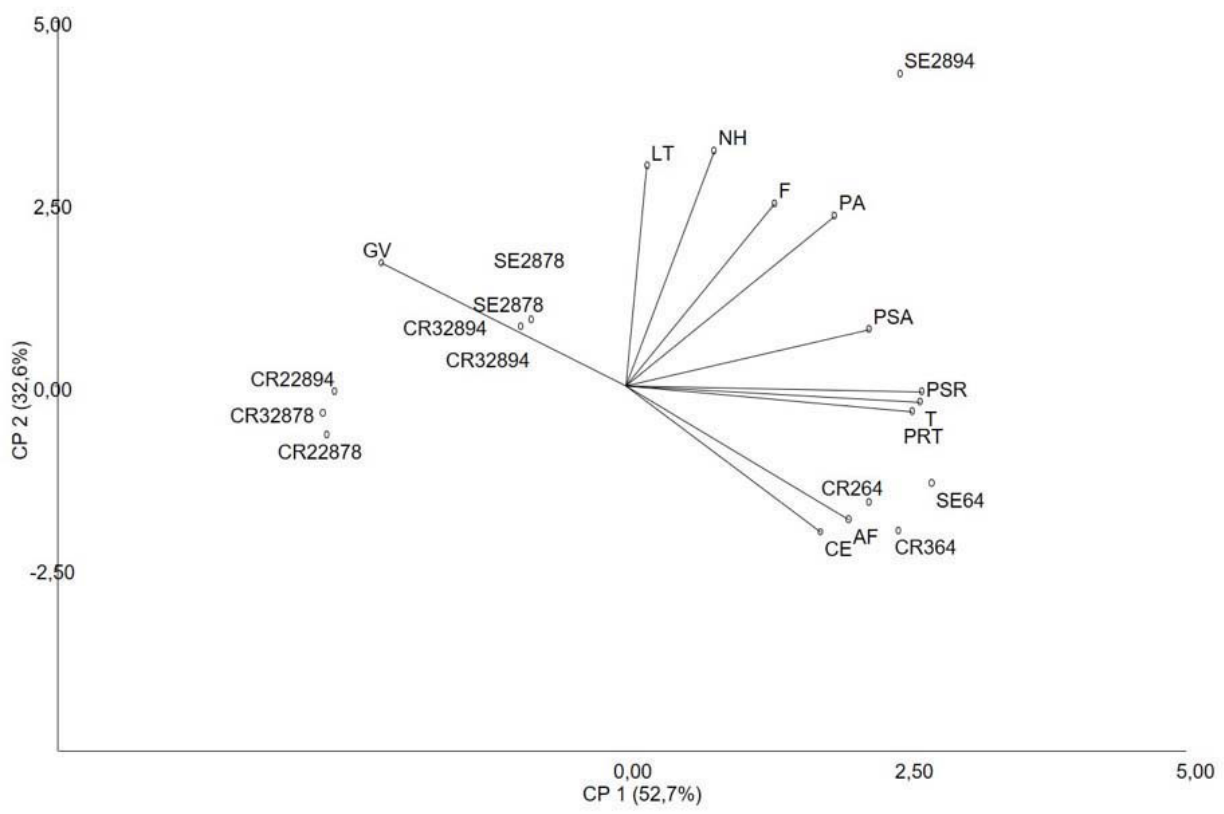

Figura 3. Biplot multivariado por componentes principales en tres clones de camote (Ipomoea batatas L.) y tres tratamientos de estrés hídrico en nueve variables evaluadas. Instituto Nacional de Investigaciones Agrícolas-Centro Nacional de Investigaciones Agropecuarias (INIA-CENIAP). Maracay, Venezuela, 2015.

GV: grado de verdor, LT: largo del tallo, NH: número de hojas, F: fotosíntesis, T: transpiración, PSA: peso seco aéreo, PSR: peso seco raíz, $\mathrm{AF}$ : área foliar, $\mathrm{CE}$ : conductancia estomática, $\mathrm{SE}$ : con riego todo el ciclo, CR2: con riego los primeros dos meses, CR3: con riego los primeros tres meses. 64, 2878 y 2894 corresponden a clones, respectivamente.

Figure 3. Multivariate biplot by main components in three sweet potato (Ipomoea batatas L.) clones and three water stress treatments in nine evaluated variables. Instituto Nacional de Investigaciones Agrícolas-Centro Nacional de Investigaciones Agropecuarias (INIACENIAP). Maracay, Venezuela, 2015.

GV: degree of greenery, LT: stem length, NH: number of leaves, F: photosynthesis, T: transpiration, PSA: aerial dry weight, PSR: root dry weight, AF: leaf area, CE: stomatal conductance, SE: with irrigation throughout the cycle, CR2: with irrigation the first two months, CR3: with irrigation the first three months. 64, 2878, and 2894 correspond to clones, respectively.

Cuadro 2. Valores promedio del peso seco de raíz (PSR), transpiración (T), grado de verdor (GV), y peso de raíz total (PRT) en tres clones de camote (Ipomoea batatas L.). Campo de Investigación Experimental del Instituto Nacional de Investigaciones AgrícolasCentro Nacional de Investigaciones Agropecuarias (INIA-CENIAP). Maracay, Venezuela, 2015.

Table 2. Average value of dry root weight (PSR), transpiration (T), greenness degree (GV), and total root weight (PRT) in three sweet potato (Ipomoea batatas L.) clones. Experimental del Instituto Nacional de Investigaciones Agrícolas-Centro Nacional de Investigaciones Agropecuarias (INIA-CENIAP). Maracay, Venezuela, 2015.

\begin{tabular}{|c|c|c|c|c|c|c|c|c|}
\hline & PSR & & $\mathbf{T}$ & & GV & & PRT & \\
\hline Clon & $\mathrm{DMS}=116,93433$ & & $\mathrm{DMS}=0,11213$ & & $\mathrm{DMS}=5,43899$ & & $\mathrm{DMS}=329,42152$ & \\
\hline 64 & $261,11 \pm 41,66$ & A & $0,43 \pm 0,17$ & A & $37,23 \pm 1,36$ & $\mathrm{~B}$ & $572,22 \pm 155,59$ & A \\
\hline 2894 & $194,44 \pm 10,23$ & $\mathrm{AB}$ & $0,32 \pm 0,09$ & B & $46,74 \pm 6,85$ & A & $306,48 \pm 367,37$ & $\mathrm{AB}$ \\
\hline 2878 & $125,69 \pm 45,80$ & B & $0,25 \pm 0,08$ & B & $46,55 \pm 4,25$ & A & $79,17 \pm 70,71$ & B \\
\hline
\end{tabular}

Medias con una letra común no son significativamente diferentes (Tukey, p>0,05) / Means with a common letter are not significantly different (Tukey, $\mathrm{p}>0.05$ ).

Promedio \pm desviación estándar / Average \pm standard deviation. 
la producción de follaje para alimentación animal, es conveniente pensar en una estrategia para manejar el recurso hídrico, ya que las diferencias fueron más del doble con relación a los tratamientos a los que se les suspendió el riego durante los primeros dos meses.

\section{Análisis multivariado por componentes principales}

En los análisis multivariados por componentes principales no es válido evaluar solo un componente para explicar la variabilidad de los datos. Así, se encontró que solo 52,7 \% sintetizó la variabilidad en el primer componente, por lo que, con base en el criterio de selección de componentes principales de Kaiser (Pla, 1986), se utilizaron los dos primeros componentes para un valor de $85 \%$. Además, se puede observar que las variables asociadas al crecimiento vegetativo, tales como número de hojas, largo del tallo y peso aéreo, están relacionadas con la variable fotosíntesis, mientras que, las variables de transpiración, conductancia estomática, peso seco de raíz y peso de raíz total, tuvieron vectores cercanos, con asociación entre estas variables con todos los tratamientos de estrés y el clon 64. Se observó que los tratamientos sin estrés alcanzaron los mayores valores de grado de verdor, por lo cual se encontró la relación de esta variable con el tratamiento sin estrés y el clon 2894 (Figura 3).

\section{Discusión}

La interacción significativa del clon 2894 con todos los tratamientos de estrés mostró los mayores valores para la longitud del tallo con crecimiento hasta tres veces más que los otros clones en todos los tratamientos de riego, por lo que se podría seleccionar este clon para validarlo a nivel comercial. Este comportamiento se debe a que el crecimiento de los brotes en el cultivo de camote continúa a pesar de la sequía, pero con hojas más pequeñas para minimizar el exceso de transpiración, tal como como ocurrió en las investigaciones de Laurie et al. (2015) y Nedunchezhiyan et al. (2012), en las que observaron diferencias significativas entre clones de camote y tratamientos de estrés hídrico, con la tendencia a sobresalir el mismo clon en todos los tratamientos de estrés durante tres años de evaluación.

La reducción en la longitud del tallo (en relación con el control con humedad a capacidad de campo en el suelo) en quince accesiones de camote expuestas al estrés por sequía varió del 16,1 al 46,0 \% en comparación con el testigo sin estrés (Deblonde \& Ledent, 2001). Estos resultados confirman el valor genético que tienen algunos cultivares para superar la condición de sequía con respecto al crecimiento vegetativo (Saraswati et al., 2004).

El número de hojas fue la única variable que mostró diferencias significativas en los tratamientos de estrés, este comportamiento lo mostró La Rosa (2008) en cultivares de camote, donde se observó que variaciones de agua en el suelo provocaron marcadas diferencias en la cobertura y en el tamaño del follaje, pero sin ninguna relación con la actividad fotosintética, ya que coberturas pequeñas llegaron a producir raíces reservantes, por lo que este es el órgano más afectado, constituyéndose como una buena referencia del estado hídrico del suelo.

La longitud del tallo en los genotipos de camote PROC 65-3 y Yellow Japanese sembrados con contenido de agua en el suelo a punto de marchitez permanente, disminuyó el valor de la longitud del tallo. En contraste con el cultivar de camote Tainung 57, sembrado en condiciones de déficit hídrico con $15 \%$ y $8 \%$ de la humedad del suelo, las cuales disminuyeron en 20,50 \% y 31,68 \% la longitud del tallo en comparación con el testigo sembrado con humedad del suelo del $100 \%$ (Yooyongwech et al., 2013), comportamiento similar al del presente estudio.

El clon 64 fue el que acumuló la mayor biomasa seca y fresca de raíz en todos los tratamientos de estrés con tasas transpiratorias superiores al resto de los clones. Este clon tuvo también las mayores tasas de fotosíntesis y por ende valores del doble en área foliar. Esto se debe a que el peso seco total por planta entre los cultivares de batata es bastante uniforme, las diferencias se observaron principalmente entre los órganos de la planta que son 
más afectados por el régimen de agua, que para la investigación de La Rosa (2008) fue la parte vegetativa. Sin embargo, la biomasa seca de hojas, pecíolos, tallos y raíces fueron mayores en las plantas en el tratamiento de 100 $\%$ de la humedad a capacidad de campo durante todas las etapas de crecimiento de las plantas, comparadas con las cultivadas al $50 \%$ de la humedad de la capacidad de campo (Zhang et al., 2015).

Las respuestas fisiológicas de las plantas al déficit de agua de los clones en el presente estudio incluyeron el marchitamiento, reducción en el área y la abscisión de las hojas, así como la estimulación del crecimiento de las raíces al dirigir los nutrientes a las partes subterráneas de las plantas (Muhammad et al., 2011). Es por ello, que la longitud promedio del tallo de $38 \mathrm{~cm}$ más larga en el estudio de Omotobora et al. (2014), se registró en el cultivar de camote Zapallo, mientras que la más corta se encontró en el período lluvioso con una longitud de 28,6 cm.

El grado de verdor es de suma importancia, ya que el fundamento de la variable se basa en las estrechas relaciones que existen entre las lecturas del medidor Spad y el contenido de clorofila y esta última, a su vez, asociada a la fotosíntesis y a los componentes de rendimiento (León et al., 2014). A pesar de ello, el clon con el mejor comportamiento agronómico (clon 64) tuvo los menores valores de Spad en comparación con los otros dos clones, lo que indica que la diferencia en este valor implicó menores concentraciones en el contenido de clorofila, sin embargo, no se vieron afectadas las variables asociadas al intercambio gaseoso y rendimiento, posiblemente debido a que este clon se encontraba en su máximo potencial de rendimiento y estaba en fases fenológicas finales, donde predomina amarillamiento de hojas y muerte de la planta por la máxima acumulación de materia seca en raíces.

El clon 64 biológicamente alcanzó las mayores tasas de fotosíntesis y produjo 572,22 $\mathrm{g} \mathrm{planta}^{-1}$ de raíces totales, lo que implica que con una densidad de siembra de 32000 plantas ha ${ }^{-1}$ alcanzó un rendimiento promedio de 18,3 t ha $^{-1}$, rendimiento seis veces mayor que el clon con el menor comportamiento y con tasa de transpiración casi del doble, lo que indica que el clon se adaptó fisiológicamente para no ser afectado por ningún tratamiento de estrés y así evitar la pérdida por transpiración, sin afectar las tasas de fotosíntesis y, por ende, el rendimiento de raíces. La razón de esto puede deberse a que los estomas de las hojas inducidos por la sequía se cierran parcialmente y disminuyen la concentración intercelular de $\mathrm{CO}_{2}$ (Chaves et al., 2002). Además, la acumulación y partición de materia seca en los clones de camote dependen del estado de crecimiento de las plantas y el contenido de humedad en el suelo y estas a su vez, por las variables de intercambio gaseoso, incluida la tasa fotosintética, tasa de transpiración, conductancia estomática y las proporciones de $\mathrm{CO}_{2}$ intercelular a las concentraciones de $\mathrm{CO}_{2}$ atmosférico (Zhang et al., 2015). Esto podría indicar que hay clones de camote que tienen la habilidad para adaptarse a niveles estresantes de humedad del suelo a través de mecanismos fisiológicos (control del cierre estomático), por lo que podrían seleccionarse como promisorios frente a estrés por sequía (Ekanayake \& Collins, 2004).

El cultivo de camote es resistente a la sequía en términos de supervivencia, debido a que en la medida que aumenta la sequía, así ocurre la resistencia estomatica para evitar pérdidas por evapotranspiración y, por ende, disminuciones en las tasas fotosintéticas (Mohammed \& Sadiq, 2016; Mukhopadhyay et al., 2011), tal como ocurrió en el presente estudio.

\section{Conclusiones}

Bajo condiciones de estrés hídrico las plantas de camote se adaptaron fisiológicamente para no perder agua por la transpiración sin sacrificar las tasas de fotosíntesis, con los cuales los clones lograron alcanzar una acumulación de biomasa aérea, raíces y rendimiento del tubérculo similar al cultivo con riego.

El uso de variables asociadas al intercambio gaseoso permitió identificar clones promisorios de camote bajo condiciones de estrés hídrico.

Se recomienda en el futuro estudiar variables asociadas a la palatabilidad, propiedades mecánicas y de texturas, reológicas, entre otras de interés para la industria en los clones de camote usados en esta investigación.

Clones de batata pueden producir semilla de calidad en períodos de estrés hídrico, de acuerdo con su genética. 


\section{Referencias}

Chaves, M. M., Pereira, J. S., Maroco, J., Rodrigues, M. L., Ricardo, C. P. P., Osório, M. L., \& Pinheiro, C. (2002). How plants cope with water stress in the field? Photosynthesis and growth. Annals of Botany, 89(7), 907-916. https://doi. org/10.1093/aob/mcf105

Confederación de Asociaciones de Productores Agropecuarios de Venezuela. (2019, 15 de Julio 2019). Producción. https:// fedeagro.org/estadisticas-agricolas/produccion-agropecuaria/produccion/

Cusumano, C., \& Zamudio, N. (2013). Manual técnico para el cultivo de batata (camote o boniato) en la provincia de Tucumán (Argentina). Ediciones Instituto Nacional de Tecnología Agropecuaria. https://inta.gob.ar/sites/default/files/scripttmp-manual_batata.pdf

Cyril, A., Guaf, E., \& Komolong, B. (2015). Screening sweetpotato (Ipomoea batatas) genotypes under soil moisture deficit condition using stress tolerance indices. Archives of Applied Science Research, 7(11), 23-29. https://www. scholarsresearchlibrary.com/abstract/screening-sweetpotato-ipomoea-batatas-genotypes-under-soil-moisture-deficitcondition-using-stress-tolerance-indices-1557.html

Deblonde, P. M. K., \& Ledent, J. F. (2001). Effects of moderate drought conditions on green leaf number, stem height, leaf length and tuber yield of potato cultivars. European Journal of Agronomy, 14(1), 31-41. http://dx.doi.org/10.1016/ S1161-0301(00)00081-2

De-Tafur, S. M. (2002). Fisiología de la yuca (Manihot esculenta Crantz) (Capítulo 3.). En H. Ceballos, \& B. Ospina (Eds). La yuca en el Tercer Milenio: Sistemas modernos de producción, procesamiento, utilización y comercialización (pp. 4-45). Centro Internacional de Agricultura Tropical.

Ekanayake, I. J., \& Collins, W. (2004). Effect of irrigation on sweet potato root carbohydrates and nitrogenous compounds. Journal of Food Agriculture and Environment, 2, 243-248. https://doi.org/10.1234/4.2004.130

Food and Agriculture Organization. (2019, 15 de Julio 2019). Estadística agropecuaria. http://www.fao.org/faostat/es/\#data/QC

Guerrero, F. (2020). Tolerancia al estrés hídrico inducido por memoria en parientes silvestres de camote (Ipomoea series Batatas (Choisy) D. F. Austin) [Tesis de Pregrado, Universidad Nacional Agraria La Molina]. Repositorio de la Universidad Nacional Agraria La Molina. http://repositorio.lamolina.edu.pe/bitstream/handle/UNALM/4419/ guerrero-zurita-fernando.pdf?sequence $=1 \&$ isAllowed $=\mathrm{y}$

La Rosa, R. (2008). Respuestas fisiológicas de camote Ipomoea batatas (L.) Lam. a diferentes frecuencias de riego [Tesis de Maestría, Universidad Nacional Mayor de San Marcos]. Repositorio de la Universidad Nacional Mayor de San Marcos. https://cybertesis.unmsm.edu.pe/bitstream/handle/20.500.12672/5334/larosa_lr.pdf?sequence=1\&isAllowed=y

Laurie, R. N., Laurie, S. M., Du Plooy, C. P., Finnie, J. F., \& Van Staden, J. (2015). Yield of drought-stressed sweet potato in relation to canopy cover, stem length and stomatal conductance. Journal of Agricultural Science, 7(1), 201-214. http://dx.doi.org/10.5539/jas.v7n1p201

León, R., Pérez, M., Fuenmayor, F., Gutiérrez, V., \& Marín, C. (2014). Caracterización ecofisiológica de cuatro clones de yuca (Manihot esculenta Crantz) en el Campo Experimental del INIA-CENIAP. Agronomía Tropical, 64(1-2), 97-105. http://ve.scielo.org/scielo.php?pid=S0002-192X2014000100010\&script=sci_abstract 
Mantovani, E. C., Delazari, F. T., Dias, L. E., de Assis, I. R., Vieira, G. H., \& Landim, F. M. (2013). Eficiência no uso da água de duas cultivares de batata-doce em resposta a diferentes lâminas de irrigação. Horticultura Brasileira, 31(4), 602-606. https://doi.org/10.1590/S0102-05362013000400015

Mohammed, S., \& Sadiq, K. A. (2016). Productivity of sweet potato (Ipomoea batatas L.) as influenced by water stress and variety in Sokoto Sudan Savannah, Nigeria. International Journal of Plant \& Soil Science, 12(1), 1-9. http://doi. org/10.9734/IJPSS/2016/25963

Muhammad, W., Ali, A., Tahir, M., Nadeem, M. A., Ayub, M., Tanveer, A., Ahmad, R., \& Hussain, M. (2011). Mechanism of drought tolerance in plant and its management through different methods. Continental Journal of Agricultural Science, 5(1), 10-25. https://doi.org/10.5281/zenodo.839955

Mukhopadhyay, S. K., Chattopadhyay, A., Chakraborty, I., \& Bhattacharya, I. (2011). Crops that feed the world 5. Sweetpotato. Sweetpotatoes for income and food security. Food Security, 3(3), 283-305. https://doi.org/10.1007/s12571-0110134-3

Nedunchezhiyan, M., Byju, G., \& Jata, S. K. (2012). Sweet potato agronomy. Fruit, Vegetable and Cereal Science and Biotechnology, 6(1), 1-10. http://www.globalsciencebooks.info/Online/GSBOnline/images/2012/FVCSB_6(SI1)/ FVCSB_6(SI1)1-10o.pdf

Omotobora, B. O., Adebola, P. O., Modise, D. M., Laurie, S. M., \& Gerrano, A. S. (2014). Greenhouse and field evaluation of selected sweetpotato (Ipomoea batatas L.) LAM) accessions for drought tolerance in South Africa. American Journal of Plant Sciences, 5(21), 3328-3339. http://dx.doi.org/10.4236/ajps.2014.521348

Pla, L. (1986). Análisis multivariado: Método de componentes principales. Secretaría General de los Estados Americanos.

Ravi, V., \& Indira, P. (1999). Crop physiology of sweet potato. Horticultural Reviews, 23, 277-338. https://doi. org/10.1002/9780470650752.ch6

Rodríguez, A. (2016). Dinámica de la respuesta del cultivo de camote (Ipomoea batatas L.) al estrés hídrico y salino [Tesis de Doctorado, Universidad Nacional Agraria La Molina]. Repositorio de la Universidad Nacional Agraria La Molina. http://repositorio.lamolina.edu.pe/bitstream/handle/UNALM/2813/F06-R6-T.pdf?sequence=4\&isAllowed=y

Saraswati, P., Johnston, M., Coventry, R., \& Holtum, J. (2004, September 26 - October 1). Identification of drought tolerant sweet potato (Ipomoea batatas (L.) Lam) cultivars [Poster Presentation]. $4^{\text {th }}$ International Crop Science Congress, Brisbane, Australia. http://www.agronomyaustraliaproceedings.org/images/sampledata/2004/poster/1/1/1400_saraswatip.pdf

Siddique, M. R. B., Hamid, A. I. M. S., \& Islam, M. S. (2000). Drought stress effects on water relations of wheat. Botanical Bulletin of Academia Sinica, 41, 35-39. https://ejournal.sinica.edu.tw/bbas/content/2000/1/bot11-06.html

Sokoto, M., \& Sadi, K.A. (2016). Productivity of sweet potato (Ipomoea batatas L.) as influenced by water stress and variety in Sokoto Sudan Savannah, Nigeria. Journal of Plant \& Soil Science, 12(1), 1-9. https://doi.org/10.9734/ IJPSS/2016/25963

Van-Heerden, P. D. R., \& Laurie, R. (2008). Effects of prolonged restriction in water supply on photosynthesis, shoot development and storage root yield in sweet potato. Physiologia Plantarum, 134(1), 99-109. http://dx.doi. $\operatorname{org} / 10.1111 /$ j.1399-3054.2008.01111.x

Yooyongwech, S., Theerawitaya, C., Samphumphuang, T., \& Cha-um, S. (2013). Water-deficit tolerant identification in sweet potato genotypes (Ipomoea batatas (L.) Lam.) in vegetative developmental stage using multivariate physiological indices. Scientia Horticulturae, 162, 242-251. https://doi.org/10.1016/j.scienta.2013.07.041 
Yooyongwech, S., Samphumphuang, T., Theerawitaya, C., \& Cha-um, S. (2014). Physio-morphological responses of sweet potato [Ipomoea batatas (L.) Lam.] genotypes to water-deficit stress. Plant Omics Journal, 7(5), 361-368.

Zhang, H., Zhu, L., Zhang, C., Ning, Y., \& Zhang, Y. (2015). Effect of water stress on dry mass accumulation and allocation in sweet potato based on stable isotope analysis. Canadian Journal of Plant Science, 95(2), 263-271. https://doi. org/10.4141/cjps-2014-170 